\title{
SOME ORTHOGONALITY PRESERVING OPERATORS
}

W. A. AL-SALAM AND A. VERMA

1. Introduction. Let $\left\{p_{n}(x)\right\}$ be a set of orthogonal polynomials (s.o.p.), i.e., $p_{0}(x), p_{1}(x), p_{2}(x), \cdots$ is a sequence of polynomials which satisfy the recurrence relation

$$
\begin{aligned}
p_{n+1}(x) & =\left(A_{n} x+B_{n}\right) p_{n}(x)-C_{n} p_{n-1}(x) \quad(n \geqq 0) \\
p_{-1}(x) & =0, \quad p_{0}(x)=1
\end{aligned}
$$

for some $A_{n}, B_{n}$ and $C_{n}$ where $A_{n} C_{n+1} \neq 0(n \geqq 0)$.

It is clear that if $\left\{p_{n}(x)\right\}$ is s.o.p. then $\left\{\alpha p_{n}(x+\beta)\right\}$ is also s.o.p. Let $E^{\beta}$ be the shift operator $E^{\beta} f(x)=f(x+\beta)$. We then note that the operator $L p_{n}(x)=\alpha p_{n}(x+\beta)$ can be written, on the set of all polynomials, as a differential operator of infinite order, namely,

$$
L=\alpha \sum_{n=0}^{\infty} \frac{\beta^{n}}{n !} D^{n}, \quad D=\frac{d}{d x} .
$$

Let us now consider the set $S$ of all differential operators

$$
J(D)=\sum_{k=0}^{\infty} a_{k} D^{k}, \quad a_{0} \neq 0,
$$

where $a_{0}, a_{1}, \cdots$ are constants independent of $x$. Thus $L \in S$. With no loss of generality we shall take in the following $a_{0}=1$.

Since $L$ is orthogonality preserving, i.e. if $\left\{p_{n}(x)\right\}$ is s.o.p. then $\left\{L p_{n}(x)\right\}$ is also s.o.p., it is of interest to see what operators in $S$ preserve the orthogonality property of all s.o.p.

We are also interested in elements of $S$ which preserve orthogonality of particular s.o.p. In this connection let an s.o.p. $\left\{p_{n}(x)\right\}$ be given. We say that $\left\{p_{n}(x)\right\}$ possesses an orthogonality preserving operator if there is $J \in S$ such that $\left\{p_{n}(x)\right\}$ and $\left\{J p_{n}(x)\right\}$ are sets of orthogonal polynomials. We shall prove below the following theorems:

Theorem 1. An operator $J \in S$ is orthogonality preserving for all sets of orthogonal polynomials if and only if $J=L$.

THEOREM 2. A set of orthogonal polynomials $\left\{p_{n}(x)\right\}$ possesses an orthogonality preserving operator if and only if $\left\{p_{n}(x)\right\}$ is one of the Meixner [1] polynomial sets, i.e., if and only if $\left\{p_{n}(x)\right\}$ is s.o.p. and has a generating relation of the form

Received by the editors February 20, 1969. 


$$
\sum_{0}^{\infty} p_{n}(x) t^{n} / n !=A(t) \exp (x H(t))
$$

where

$$
A(t)=1+\alpha_{1} t+\alpha_{2} t^{2}+\cdots, \quad H(t)=t+h_{1} t^{2}+h_{2} t^{3}+\cdots .
$$

Sheffer [2] proved that (1.4) holds if and only if there is an operator $B(D)$ of the form $B(D)=\sum_{\mathbf{k}=0}^{\infty} b_{k} D^{k+1}, b_{0} \neq 0$ such that

$$
B(D) p_{n}(x)=n p_{n-1}(x) \text {. }
$$

He calls such sets "sets of $A$-type 0. "

2. Proof of Theorems 1 and 2. Let $J \in S$ where $J(D)=\sum_{0}^{\infty} a_{k} D^{k}$, $a_{0} \neq 0$. Let us assume that $\left\{p_{n}(x)\right\}$ and $\left\{J p_{n}(x)\right\}$ are sets of orthogonal polynomials. We then have

$$
p_{n}(x)=\left(x-\alpha_{n}\right) p_{n-1}(x)-\beta_{n} p_{n-2}(x),
$$

and

$$
J p_{n}(x)=\left(x-\bar{\alpha}_{n}\right) J p_{n-1}(x)-\bar{\beta}_{n} J p_{n-2}(x) .
$$

If we operate on (2.1) by $J$ we get

$$
J^{\prime}(D) p_{n-1}(x)=\left(\alpha_{n}-\bar{\alpha}_{n}\right) J p_{n-1}(x)+\left(\beta_{n}-\bar{\beta}_{n}\right) J p_{n-2}(x) .
$$

Comparing coefficients of $x^{n-1}$ and $x^{n-2}$ in (2.3) we get

$$
\alpha_{n}-\bar{\alpha}_{n}=a_{1} \text { and } \beta_{n}-\bar{\beta}_{n}=(n-1)\left(2 a_{2}-a_{1}^{2}\right) \text {. }
$$

Hence (2.3) may be written in the form

$$
\bar{B}(D) p_{n}(x)=n\left(2 a_{2}-a_{1}^{2}\right) p_{n-1}(x), \quad(n \geqq 0),
$$

where $\bar{B}(t)=J^{\prime}(t) / J(t)-a_{1}$.

We now consider the two cases:

Case (i). If $2 a_{2}=a_{1}{ }^{2}$, it follows that $J^{\prime}(D) p_{n}(x)=a_{1} J(D) p_{n}(x)$ which implies that $J(t)=\mathrm{c} \exp \left(a_{1} t\right), c$ an arbitrary constant. Thus $J(D)$ is the operator (1.2).

Case (ii). If $2 a_{2}-a_{1}^{2}=b_{0} \neq 0$. We may write (2.4) as

$$
B(D) p_{n}(x)=b \bar{B}(D) p_{n}(x)=n p_{n-1}(x) \quad(n \geqq 0)
$$

where $b=1 / b_{0} \neq 0$ and

$$
B(D)=\sum_{k=0}^{\infty} b_{k} D^{k+1}, \quad b \neq 0 .
$$


Hence, $\left\{p_{n}(x)\right\}$ is of Sheffer $A$-type 0 [2] and must satisfy (1.5) with $H(t)$ defined by $H(B(t))=t$.

This finishes the proof of Theorem 1 and we only need to prove that if $\left\{p_{n}(x)\right\}$ is one of the orthogonal sets of polynomials and is of $A$-type 0 (i.e., satisfies (1.4)) then $\left\{p_{n}(x)\right\}$ possesses an orthogonality preserving operator. Indeed let $\left\{p_{n}(x)\right\}$ satisfy (1.5) and $J(t)$ be the solution of the differential equation $J^{\prime}(t)=\gamma B(t) J(t), J(0)=1$ and $\gamma$ is arbitrary. Thus we must have

$$
J^{\prime}(D) p_{n}(x)=\gamma n J(D) p_{n-1}(x) .
$$

Hence we obtain from (2.1) and (2.5) that

$$
J p_{n}(x)=\left(x-\alpha_{n}\right) J p_{n-1}(x)-\left[\beta_{n}-(n-1) \gamma\right] J p_{n-2}(x) \quad(n \geqq 1)
$$

which is of the form (1.1). Hence $\left\{J_{p_{n}}(x)\right\}$ is a set of orthogonal polynomials.

Meixner [1] and Sheffer [2] determined all orthogonal polynomials which are of $A$-type 0 . We give them below together with their orthogonality preserving operator $J(D)$ and evaluate $J p_{n}(x)$.

(i) The Laguerre polynomials $L_{n}^{(\alpha)}(x)$.

so that

$$
H(t)=B(t)=t /(t-1), \quad J(t)=(1-t)^{\gamma},
$$

$$
(1-D)^{\gamma} L_{n}^{(\alpha)}(x)=L_{n}^{(\alpha+\gamma)}(x)
$$

which is valid for arbitrary $\gamma$.

(ii) The Hermite polynomials $H_{n}(x) . H(t)=2 t, B(t)=t / 2$ so that $J(t)=\exp \left(\gamma t^{2}\right)$ where $\gamma$ is an arbitrary constant. We have

$$
\exp \left(\gamma D^{2}\right) H_{n}(x)=(1-4 \gamma)^{-n / 2} H_{n}\left(x(1-4 \gamma)^{1 / 2}\right) .
$$

(iii) The Charlier polynomials $c_{n}(x ; a) . H(t)=\log (1-t / a), B(t)$ $=a\left(1-e^{t}\right)$ so that $J(t)=\exp \left(-\gamma\left(1-e^{t}\right)\right)$ where $\gamma$ is an arbitrary constant.

The relation

$$
e^{\gamma \Delta} c_{n}(x ; a)=(1+\gamma / a)^{n} c_{n}(x ; a+\gamma)
$$

can be easily verified.

(iv) The Meixner polynomials $M_{n}(x ; \mu, d)$ defined by means of

$$
\begin{gathered}
\frac{(1-\mu t)^{x+d}}{(1-t)^{x}}=\sum_{n=0}^{\infty} M_{n}(x ; \mu, d) t^{n} / n !, \\
H(t)=\log \frac{1-\mu t}{1-t}, \quad B(t)=\frac{e^{t}-1}{e^{t}-\mu} .
\end{gathered}
$$


As before we have $J(t)=e^{\gamma t}\left(e^{t}-\mu\right)^{\gamma(\mu-1)}$ where $e^{\gamma}$ is an arbitrary constant. Hence we have

$$
\begin{aligned}
& E^{\gamma}(E-\mu)^{\gamma(\mu-1)} M_{n}(x ; \mu, d) \\
& \quad=(1-\mu)^{\gamma(\mu-1)} M_{n}(x+\gamma \mu ; \mu, d+\gamma-\gamma \mu) .
\end{aligned}
$$

In the above examples $E$ is the shift operator $E f(x)=f(x+1)$ and $\Delta$ is the difference operator $\Delta=E-1$. We also note that $j(D)$ was determined up to a shift operator because if $J(D)$ is orthogonality preserving then $E^{c} J(D)$ has the same property. Thus we shall consider two operators $J_{1}(D)$ and $J_{2}(D)$ equal if there is a constant $c$ such that $J_{1}(D)=E^{c} J_{2}(D)$.

\section{REFERENCES}

1. J. Meixner, Orthogonale Polynomsysteme mit einer besonderen Gestalt der erzeugenden Funktion, J. London Math. Soc. 9 (1934), 6-13.

2. I. M. Sheffer, Some properties of polynomials of type zero, Duke Math. J. 5 (1939), 590-622.

UNIVERSITY OF ALBERTA 